\title{
PA-MSHA inhibits proliferation and induces apoptosis in human non-small cell lung cancer cell lines with different genotypes
}

\author{
XINMIN ZHAO ${ }^{1,2}$, XIANGHUA WU ${ }^{1,2}$, WEN YU ${ }^{2,3}$, XUWEI CAI $^{2,3}$, QI LIU $^{2,3}$, XIAOLONG FU $^{2,3}$, \\ ZHEBIN LIU ${ }^{2,4}$, DALI HU ${ }^{5}$, SHIYUN PAN ${ }^{5}$ and QILING HUANG ${ }^{5}$ \\ ${ }^{1}$ Department of Medical Oncology; ${ }^{2}$ Fudan University Shanghai Cancer Center, Department of Oncology, \\ Shanghai Medical College, Fudan University, Shanghai 200032; Departments of ${ }^{3}$ Radiation Oncology, \\ ${ }^{4}$ Breast Surgery and ${ }^{5}$ Medicine, Beijing Wonder Biopharmaceutical Co., Ltd., Beijing 101407, P.R. China
}

Received July 17, 2015; Accepted July 26, 2016

DOI: $10.3892 / \mathrm{mmr} .2016 .5869$

\begin{abstract}
The present study examined the potential of Pseudomonas aeruginosa-mannose sensitive hemagglutinin (PA-MSHA) to inhibit proliferation and induce apoptosis in non-small-cell lung cancer (NSCLC) cell lines. It also investigated its mechanisms of action in different genotypes of human NSCLC. A total of three NSCLC cell lines, PC-9, A549, and NCI-H1975, were treated with PA-MSHA at different concentrations. The anti-proliferative effect of PA-MSHA was evaluated using a 3-(4,5-dimethylthiazol-2-yl)-2,5-diphenyltetrazolium bromide assay. Cell cycle distribution and apoptosis induced by the treatment were measured by flow cytometry (FCM) with Annexin V/propidium iodide staining. Western blotting was conducted to determine the expression level of apoptosis-associated proteins. PA-MSHA was demonstrated to exert a time- and concentration-dependent cytotoxic effect in PC-9, A549, and NCI-H1975 cells. The FCM indicated that all the different concentrations of PA-MSHA used in the present study induce apoptosis and cell cycle arrest of NSCLC cells. Treatment with PA-MSHA may exert anti-proliferative effects on NSCLC cells by affecting regulation of the cell cycle and inducing apoptosis that is mediated in part by an intrinsic apoptosis signaling pathway. These data suggest that PA-MSHA has the potential to inhibit proliferation and induce apoptosis in NSCLC cells. Furthermore, these data
\end{abstract}

Correspondence to: Dr Xiaolong Fu, Fudan University Shanghai Cancer Center, Department of Oncology, Shanghai Medical College, Fudan University, 270 Dong An Road, Shanghai 200032, P.R. China E-mail: xlfu1964@hotmail.com

\begin{abstract}
Abbreviations: PA-MSHA, Pseudomonas aeruginosa-mannose sensitive hemagglutinin; NSCLC, non-small-cell lung cancer; MTT, 3-(4,5-dimethylthiazol-2-yl)-2,5-diphenyltetrazolium bromide; FCM, flow cytometry; PI, propidium iodide; OS, overall survival; EGFR, epidermal growth factor receptor; TKIs, tyrosine kinase inhibitors
\end{abstract}

Key words: NSCLC, EGFR TKI, resistance, PA-MSHA, apoptosis provide mechanistic details for the potential application of PA-MSHA-based therapeutic strategies for the treatment of different NSCLC genotypes. This present study suggests potential novel strategies to maximize effective therapeutic strategies targeting anti-epidermal growth factor receptor for future clinical trials.

\section{Introduction}

Lung cancer was the most common type of cancer in men and women in 2012, and it continues to be the most common cause of cancer-associated mortality. Lung cancer is expected to account for $>25 \%$ of male and female cancer deaths, although there has been a slight decrease in mortality and incidence (1). Approximately $85 \%$ of lung cancer patients have non-small cell lung cancer (NSCLC). In early stage NSCLC, adjuvant chemotherapy is effective at improving patient disease-free survival and overall survival (OS). For advanced stage NSCLC, chemotherapy is the primary first-line treatment, however, the response rate is only $\sim 30 \%$, and the median OS of metastatic NSCLC is $\sim 12$ months (2).

The epidermal growth factor receptor (EGFR) is a proto-oncogene regulating cell proliferation, metastasis, and angiogenesis (3). Abnormalities in EGFR induce a marked oncogenic potential in NSCLC (4). Tyrosine kinase inhibitors (TKIs) specific to EGFR are used in second-line and even first-line therapy in patients with metastatic NSCLC, however, use of this treatment is limited by the EGFR gene mutation status (5). Gefitinib and erlotinib are first-generation EGFR TKIs, which block the EGFR signaling pathway via reversible binding to EGFR (6). In patients with EGFR mutations, including the exon 19 in-frame deletion or exon 21 L858R point mutation, the initial response to first-generation EGFR TKIs is $\sim 80 \%$. However, almost all patients acquire resistance to these agents. In $50 \%$ of these patients, resistance is derived by the occurrence of a secondary T790M mutation in exon 20 of EGFR (7). Second-generation EGFR TKIs that inhibit EGFR activity by irreversibly binding to EGFR have been clinically developed and have indicated promising anti-tumor activity in NSCLC (8). However, these irreversible EGFR TKIs are $>100$-fold less potent in NSCLC cells with the EGFR T790M mutation than in NSCLC cells 
with the EGFR exon 19 in-frame deletion mutation $(9,10)$. A previous clinical study also demonstrated a limitation of these agents that suggested the necessity for developing a novel strategy to conquer the resistance to EGFR TKIs in NSCLC (11).

It has been clearly established in the last few decades that chronic bacterial infections may contribute to carcinogenesis. Conversely, another application of bacteria and bacteria-derived products is their use to protect human beings from various malignant diseases. It is well known that bacteria mediate antitumor activities not only by indirect immune activation but also by direct tumoricidal effects (12-14). For example, genetically modified strains of Salmonella typhimurium A1-R, which is auxotrophic for Leu-Arg and has high anti-tumor virulence, is able to infect tumor cells and directly result in destruction of the nucleus. This bacterium has been successfully used to eradicate metastases in orthotopic models of prostate, breast, and pancreatic cancer, following local and systemic administration (15-18). Another important example of bacterial anti-tumor action is Streptococcus. pyogenes, which binds to target cells via fibronectin or collagen. Such direct tumor cell contact is necessary for $S$. pyogenes infection and leads to induction of the apoptotic process in tumor cells (19). A single application of live $S$. pyogenes into established pancreatic tumors resulted in complete tumor regression. Side effects included marked leukocyte infiltration and elevation of pro-inflammatory cytokines, however, $S$. pyogenes also exhibited direct lytic activity against tumor cells (19).

Pseudomonas aeruginosa injection is a type of therapeutic biological product approved in China for adjuvant treatment of patients with malignant tumors. This product is made from an inactivated mutant strain of $P$. aeruginosa (PA-MSHA) that is characterized by rich mannose-sensitive hemagglutination pili (type 1 fimbriae). PA-MSHA has been successfully used in clinical cancer therapy for a number of years, although its detailed mechanism of action remains to be elucidated. In previous studies, PA-MSHA has been demonstrated to directly inhibit tumor cell proliferation in vitro and induce apoptosis in human hepatocarcinoma, nasopharyngeal cancer and breast cancer cells $(20,21)$. Notably, an in-depth study by Liu et al (22) demonstrated that the mannose-mediated EGFR signaling pathway was involved in the apoptosis of MDA-MB-231HM and MDA-MB-468 breast cancer cells and that it was induced by PA-MSHA (22). These results suggest the potential therapeutic value of PA-MSHA in tumors typically associated with overexpression and mutation of EGFR.

In the present study, the direct tumoricidal effect of PA-MSHA on NSCLC cell lines was tested to evaluate whether $P$. aeruginosa injection is a possible adjuvant tool for NSCLC treatment, particularly in patients with EGFR TKI resistance. The three NSCLC cell lines were selected for their different gene expression statuses, as follows: i) A549, an EGFR wild-type cell line with primary EGFR TKI resistance; ii) PC-9, an EGFR TKI-sensitive cell line with the exon 19 deletion mutation; and iii) NCI-H1975, an acquired EGFR TKI-resistant cell line with the T790M mutation. The cell growth inhibition, apoptosis induction, and cell cycle redistribution of these three cell lines in response to
PA-MSHA was observed to investigate the potential of PA-MSHA in treating NSCLC resistant to EGFR TKIs.

\section{Materials and methods}

Cell lines, materials, and antibodies. Human non-small-cell lung cancer cell lines (PC-9, A549, NCI-H1975) and the BEAS-2B normal lung epidermal tissue cell line were used in the current study. The PC-9 cell line has a high sensitivity for EGFR-TKIs and an exon 19 deletion, A549 is a primary cell line resistant to EGFR-TKIs with wild-type EGFR, and NCI-H1975 has an acquired resistance to EGFR-TKIs with T790M (exon 20) and L858R (exon 21) point mutations. All cell lines were obtained from the American Type Culture Collection (Manassas, VA, USA) and cultured in DMEM medium (Gibco; Thermo Fisher Scientific, Inc., Waltham, MA, USA) supplemented with $10 \%$ heat-inactivated $\left(56^{\circ} \mathrm{C}\right.$ for $30 \mathrm{~min}$ ) fetal calf serum (GE Healthcare Life Science, Chalfont, UK), 2 mmol/1 glutamine from Gibco (Thermo Fisher Scientific, Inc.), penicillin (100 U/ml) and streptomycin $(100 \mu \mathrm{g} / \mathrm{ml})$. The cell culture was maintained in a humidified atmosphere at $37^{\circ} \mathrm{C}$ with $5 \% \mathrm{CO}_{2}$.

The strain of PA-MSHA used in the present study was kindly provided by Beijing Wanter Biopharmaceutical Co., Ltd. (Beijing, China). The PA-MSHA was scale-cultured at $37^{\circ} \mathrm{C}$ for $24 \mathrm{~h}$, inactivated using a chemical method and purified by centrifugation. The following primary antibodies used (all from Cell Signaling Technology, Inc., Danvers, MA, USA): Rabbit polyclonal anti-caspase 3 (cat. no. 9662), mouse monoclonal anti-caspase 8 (cat. no. 9746), rabbit polyclonal anti-caspase 9 (cat. no. 9502), and rabbit monoclonal anti-GAPDH (cat. no. 2118).

Cell proliferation. The effects of PA-MSHA on the survival of NSCLC cells were determined using a 3-(4,5-dimethylthiazol-2-yl)-2,5-diphenyltetrazolium bromide (MTT) assay. Cells were seeded in 96 -well plates $\left(1 \times 10^{4}\right.$ cells/well $)$ to be treated in a concentration- or time-dependent manner. Various concentrations of PA-MSHA (10, 5, 2.5, 1.25, 0.625, 0.313 and $0.156 \times 10^{9} / \mathrm{ml}$ ) were then added to BEAS-2B, PC-9, A549 and NCI-H1975 cells for different time points $(0,24$, 48, 72, 96, 120 and $144 \mathrm{~h}$ ), followed by the addition of MTT for another $4 \mathrm{~h}$. Following the removal of the culture medium, the remaining MTT formazan crystals were dissolved with dimethyl sulfoxide and measured at $490 \mathrm{~nm}$ with a microplate reader. The percentage of inhibition was calculated as follows: Inhibition ratio $(\%)=\left(1-\mathrm{OD}_{\text {sample }} / \mathrm{OD}_{\text {control }}\right) \times 1$ $00 \%$. Experiments were conducted in triplicate, and the half maximal inhibitory concentration $\left(\mathrm{IC}_{50}\right)$ values were determined.

Flow cytometry with Annexin V-fluorescein isothiocyanate and propidium iodide (PI) staining. Cells $\left(10^{6} / \mathrm{ml}\right)$ were seeded in 6-well plates and allowed to reach $70-80 \%$ confluence following $6 \mathrm{~h}$ in culture. Without changing the FBS-supplemented media, cells were treated with the indicated concentration of PA-MSHA $(0.156,0.313,0.625$, and $1.25 \times 10^{9} / \mathrm{ml}$ ) for $12 \mathrm{~h}$. Cells were then assessed by Annexin V-PI dual staining assay according to the manufacturer's protocol. Stained cells were analyzed by fluorescence 
activating cell sorting (BD Biosciences, Franklin Lakes, NJ, USA), and the percentage of apoptotic cells was determined using the ModFit LT 3.0 software (Verity Software House Inc., Topsham, ME, USA).

Western blot analysis. Cells were lysed in lysis buffer containing $2 \mathrm{M} \mathrm{NaCl}, 10 \% \mathrm{NP}-40,10 \%$ SDS, $1 \mathrm{M}$ Tris-Cl, $1 \mathrm{~g} / 1$ phenylmethylsulfonyl fluoride, $0.1 \mathrm{~g} / 1$ aprotinin and $0.01 \mathrm{~g} / 1$ leupeptin. Protein samples were quantified using the bicinchoninic acid method (cat. no. 23227; Thermo Fisher Scientific, Inc.). Samples (40 $\mu \mathrm{g} / 20 \mu \mathrm{l})$ were loaded for $10 \%$ SDS-PAGE electrophoresis and then blotted onto polyvinylidene fluoride membranes. Subsequently, the membrane was blocked with bovine serum albumin (Thermo Fisher Scientific, Inc.) for $1 \mathrm{~h}$ and the expression of the target proteins was detected using primary antibodies $(1: 1,000)$. After washing in Tris-buffered saline containing $0.05 \%$ Tween-20 the membranes were incubated with horseradish peroxidase-conjugated horse anti-mouse (cat. no. 7076) and goat anti-rabbit (cat. no. 7074) immunoglobulin G secondary antibodies (1:800; Cell Signaling Technology, Inc.). Pierce Enhanced Chemiluminescence (ECL) Western Blotting Substrate (cat. no. 32209; Thermo Fisher Scientific, Inc.) was used to detect the protein bands, and digital imaging was conducted using the Thermo Scientific myECL Imager (Thermo Fisher Scientific, Inc.).

Statistical analysis. All data are presented as the mean \pm standard deviation of at least three experiments. The statistical analysis was performed using SPSS 13.0 software for Windows (SPSS, Inc., Chicago, IL, USA). The significance of differences between experimental conditions was determined using the two-tailed Student's t-test. $\mathrm{P}<0.05$ was considered to indicate a statistically significant difference.

\section{Results}

PA-MSHA inhibits growth of NSCLC cell lines and $I_{50}$ values of PA-MSHA differ in NSCLC lines. The MTT assay demonstrated that PA-MSHA treatment had concentrationand time-dependent cytotoxic effects on A549, PC-9, and NCI-H1975 cells, however, not on BEAS-2B cells, which served as the normal control. The $\mathrm{IC}_{50}$ values are presented in Table I. As shown in Fig. 1, exposure of tumor cells to PA-MSHA for up to $144 \mathrm{~h}$ had a cumulative effect on A549 (Fig. 1A and B), PC-9 (Fig. 1C and D), and NCI-H1975 (Fig. 1E and F) cell proliferation in a concentration- and time-dependent manner. This effect was not observed in BEAS-2B cells (Fig. 1G and H). All three NSCLC cell lines were sensitive to PA-MSHA, however, it was not effective in the BEAS-2B normal lung tissue cell line. Thus, in the following experiments, the three NSCLC cell lines were focused on.

PA-MSHA results in a redistribution of the cell cycle. The underlying mechanism by which PA-MSHA exerted its anti-proliferative effects was investigated in the present study. Cells exposed to either PBS or PA-MSHA for $12 \mathrm{~h}$ were stained with PI and analyzed by flow cytometry. The A549, PC-9, and NCI-H1975 cells were exposed to
Table I. $\mathrm{IC}_{50}$ values of Pseudomonas aeruginosa-mannose sensitive hemagglutinin in different non-small cell lung cancer cell lines.

\begin{tabular}{lccc}
\hline & \multicolumn{3}{c}{$\mathrm{IC}_{50}\left(\times 10^{9} / \mathrm{ml}\right)$} \\
\cline { 2 - 4 } Cell line & $24 \mathrm{~h}$ & $48 \mathrm{~h}$ & $72 \mathrm{~h}$ \\
\hline PC-9 & 2.391 & 1.183 & 0.870 \\
A549 & 2.463 & 1.334 & 0.922 \\
NCI-H1975 & 0.963 & 0.713 & 0.140 \\
BEAS-2B & 22.565 & 16.738 & 6.456 \\
\hline
\end{tabular}

$\mathrm{IC}_{50}$, half maximal inhibitory concentration.

increasing concentrations of PA-MSHA (0.156, 0.313, 0.625, and $1.25 \times 10^{9} / \mathrm{ml}$ ), this was demonstrated to arrest the A549, PC-9 and NCI-H1975 cells in the G0/G1 phase of the cell cycle, in a dose-dependent manner, leading to a reduction in the proportion of cells in the S phase. In the PC-9 and NCI-H1975 cells, a reduction of cells in the G2/M phase was observed. In all cell lines, an additional accumulation in the sub-G1 phase was also observed (Fig. 2).

PA-MSHA induces apoptosis. Treatment with PA-MSHA was demonstrated to induce early and late apoptosis in A549, PC-9 and NCI-H1975 cells based on flow cytometric analysis. The percentage of apoptotic cells was $0.03 \%$ in the A549 cell control group (Fig. 3Aa), this was slightly elevated following exposure to PA-MSHA $\left(0.156 \times 10^{9} / \mathrm{ml}\right.$; Fig. 3Ab). The number of apoptotic cells increased in a dose-dependent manner following treatment with high concentrations of PA-MSHA $\left(0.313,0.625\right.$, and $\left.1.25 \times 10^{9} / \mathrm{ml}\right)$, the percentage of apoptotic cells was $3.30,8.40$, and $26.08 \%$ respectively $(\mathrm{P}<0.05$; Fig. 3Ac). The percentage of apoptotic cells was $6.9 \%$ in control PC-9 cells (Fig. 3Af), and treatment with PA-MSHA $\left(0.156 \times 10^{9} / \mathrm{ml}\right)$ slightly increased the amount (Fig. 3Ag). High concentrations of PA-MSHA (0.313, 0.625 , and $1.25 \times 10^{9} / \mathrm{ml}$ ) increased the number of apoptotic cells in a dose-dependent manner, the percentage of apoptotic cells was $8.83,11.85$, and $25.78 \%$, respectively $(\mathrm{P}<0.05$; Fig. 3 Ah-j). Control NCI-H1975 cells indicated an apoptosis rate of $0.39 \%$ (Fig. 3Ak), which was increased by $0.156 \times 10^{9} / \mathrm{ml}$ PA-MSHA treatment (Fig. $3 \mathrm{Al}$ ). High concentrations of PA-MSHA $\left(0.313,0.625\right.$, and $\left.1.25 \times 10^{9} / \mathrm{ml}\right)$ resulted in a dose-dependent increase in the number of apoptotic cells, the percentage of apoptotic cells was 11.04, 13.69, and $21.11 \%$, respectively $(\mathrm{P}<0.05$; Fig. 3Am-o). Following treatment with PA-MSHA, the three cell lines exhibited apoptosis in a dose-dependent manner from $0.156 \times 10^{9} / \mathrm{ml}$ (Fig. 3B).

PA-MSHA induced apoptosis via caspase cascade proteins. A549, PC-9 and NCI-H1975 cells were treated with PA-MSHA to investigate the underlying mechanism of apoptosis induction and assess the involvement of caspase-associated proteins. The lysates were analyzed using antibodies directed against caspases 3, 8, and 9 and the 
A

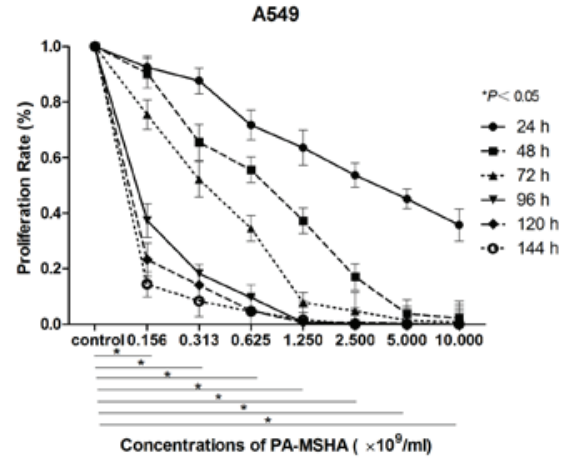

C

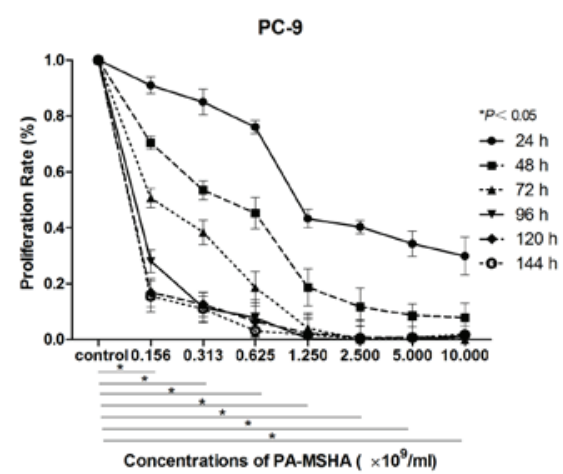

$\mathbf{E}$

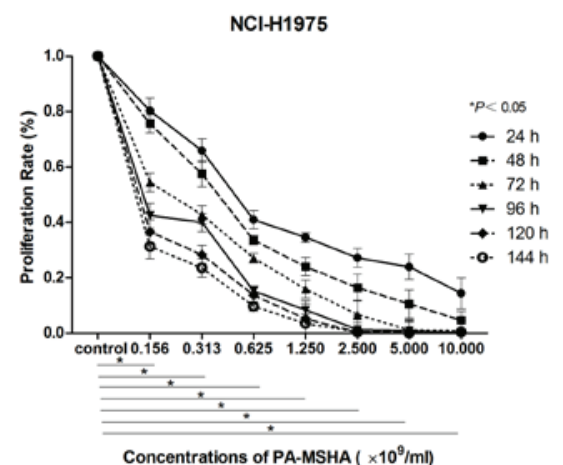

G

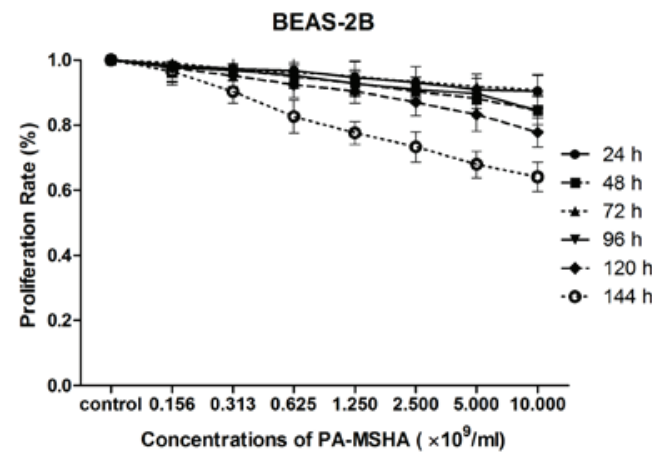

B

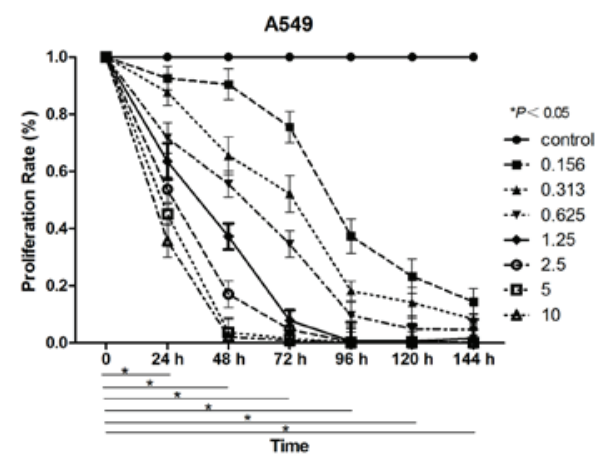

D

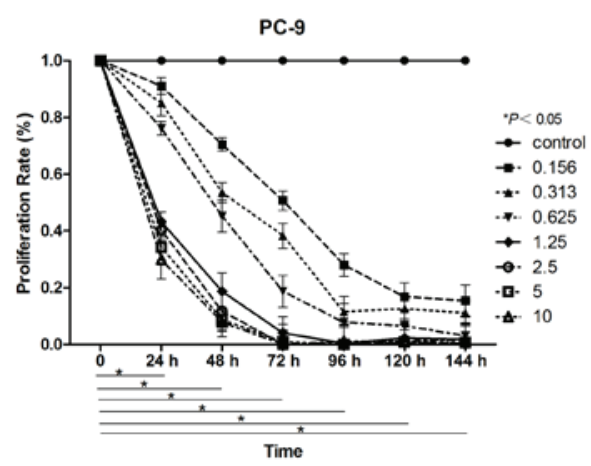

$\mathbf{F}$

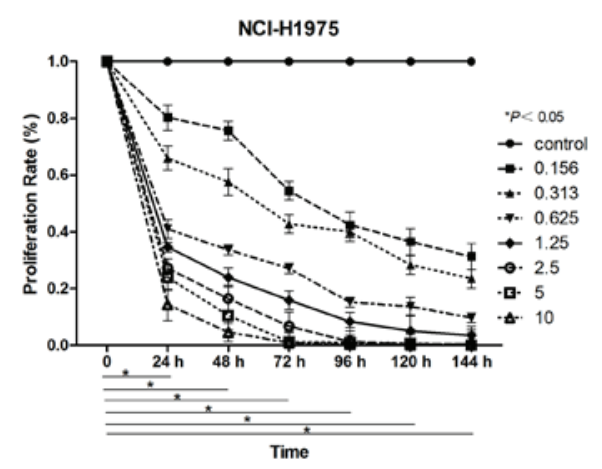

$\mathbf{H}$

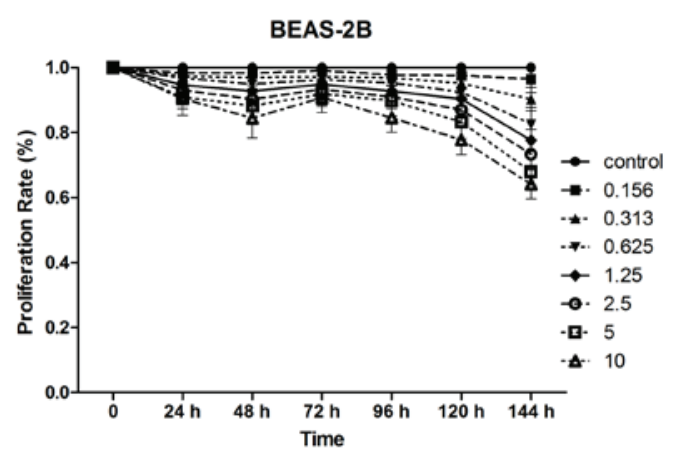

Figure 1. Effect of PA-MSHA on cell proliferation. The data are presented as the mean of triplicate results from a representative experiment, bars indicate the standard deviation. The effect of PA-MSHA on cell proliferation in (A and B) A549, (C and D) PC-9, (E and F) NCI-H1975 and (G and H) BEAS-2B cells was demonstrated to be (A, C, E and G) concentration-dependent and (B, D, F and H) time-dependent. P<0.05 for A549, PC-9, NCI-H1975 cells treated with PA-MSHA vs. the control group. PA-MSHA, Pseudomonas aeruginosa-mannose sensitive hemagglutinin.

cleaved forms of the caspase protein. When A549, PC-9, and NCI-H1975 cells were exposed to PA-MSHA for $>24 \mathrm{~h}$, there was a concentration-dependent increase in the expression levels of cleaved caspase 3 and caspase 9 proteins (Fig. 4), 
A

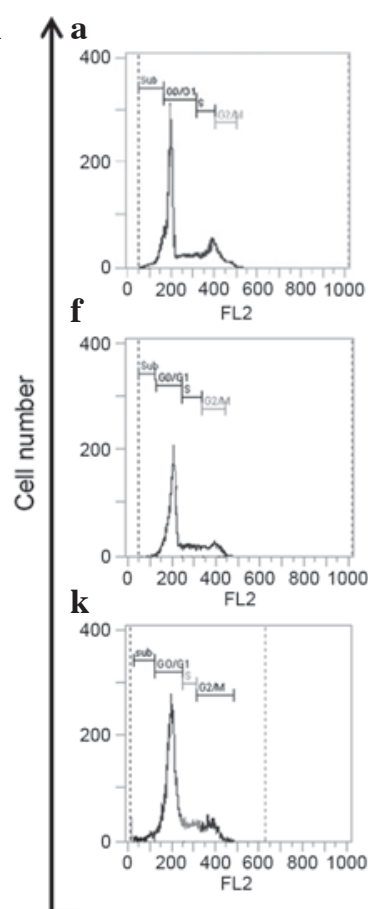

b

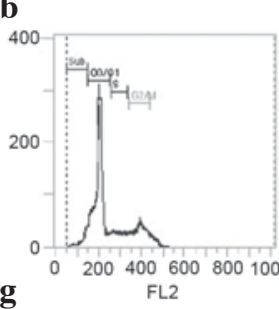

g

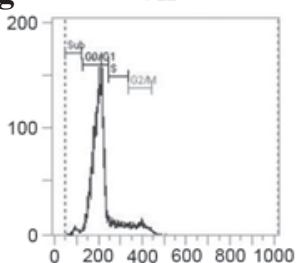

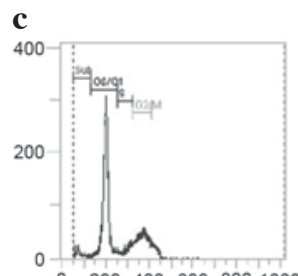

d

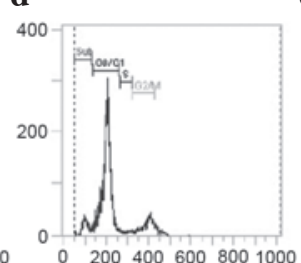

h $\begin{array}{ccc}0 & 2004006008001000 & \text { FL2 }\end{array}$
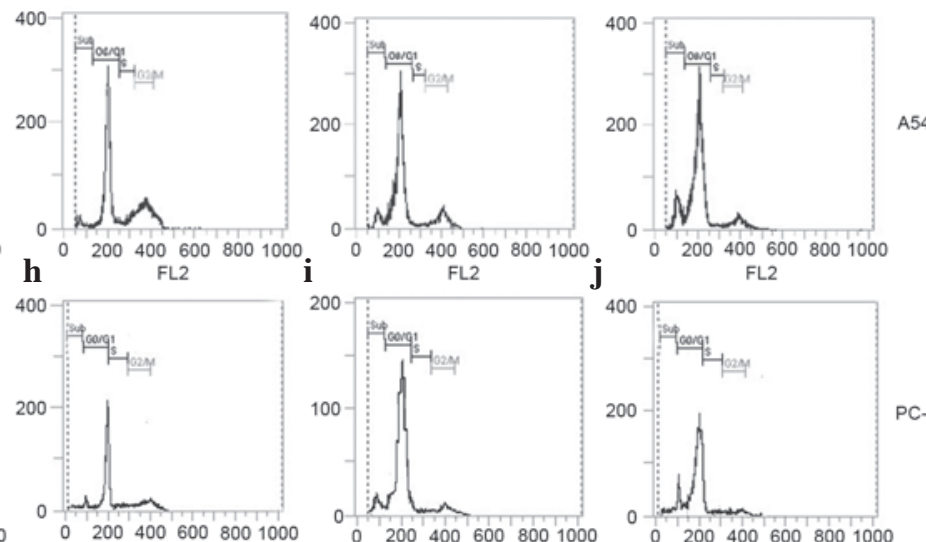

02004006008001000
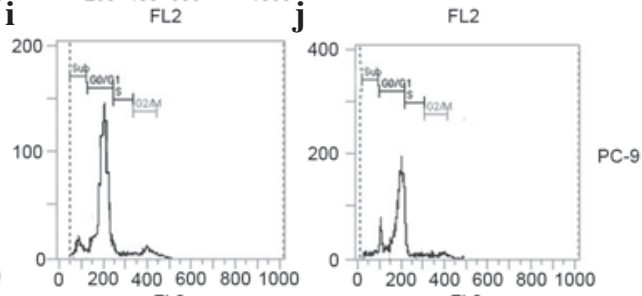

$\mathbf{m}$

FL2
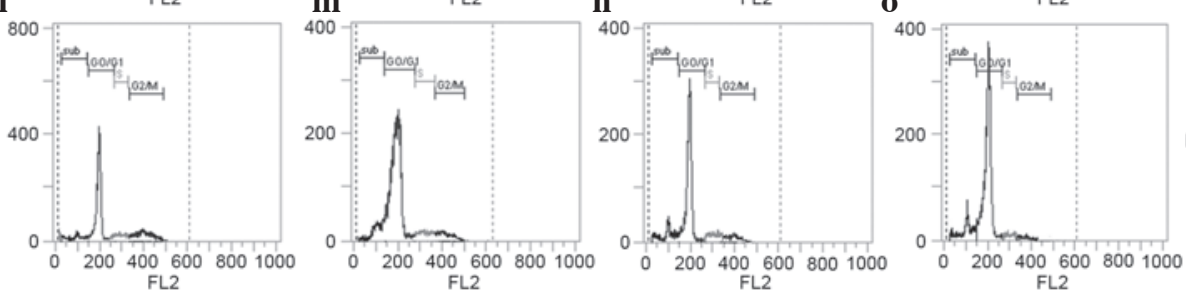

$\mathrm{NCl}-\mathrm{H} 1975$

DNA contents

0.313

0.625

1.25

Concentration of PA-MSHA $\left(\times 10^{9} / \mathrm{ml}\right)$

B

a

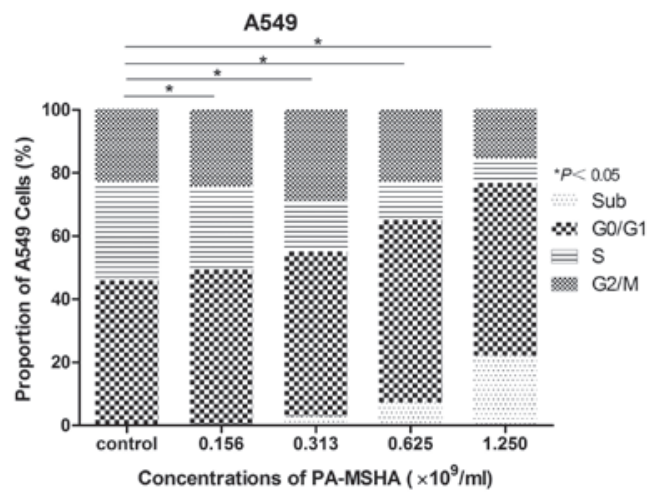

b

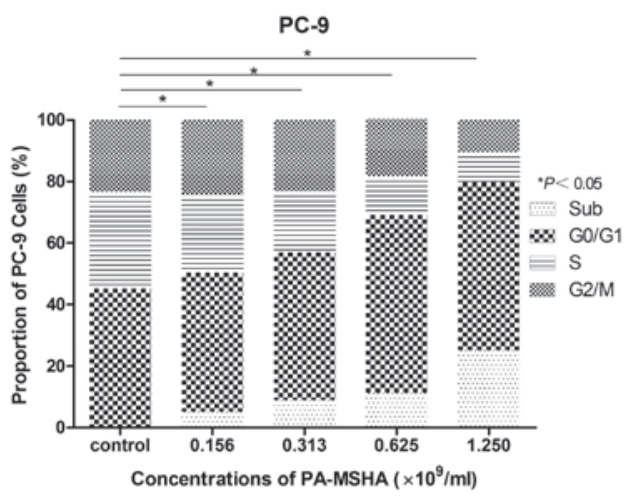

c

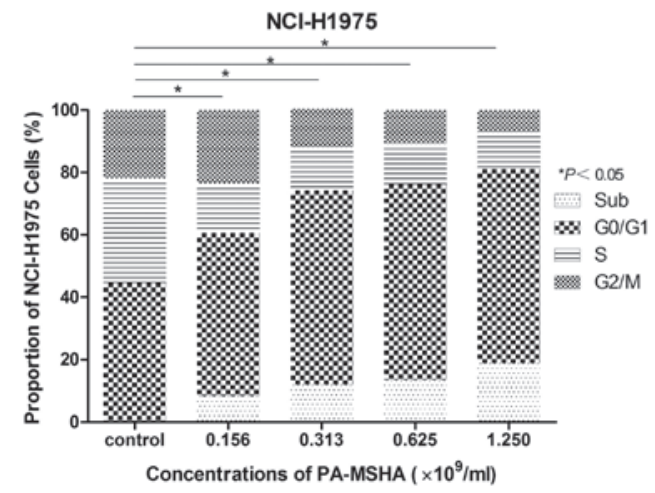

Figure 2. (A) Effect of PA-MSHA on the cell-cycle redistribution of (a-e) A549, (f-j) PC-9, and (k-o) NCI-H1975 cells at different concentrations as follows: (a, $\mathrm{f}$ and $\mathrm{k}$ ) $0,\left(\mathrm{~b}, \mathrm{~g}\right.$ and l) $0.156,(\mathrm{c}, \mathrm{h}$ and $\mathrm{m}) 0.313$, (d, i and n) 0.625 and $\left(\mathrm{e}, \mathrm{j}\right.$ and o) $1.25 \times 10^{9} / \mathrm{ml}$. The percentage of cells in each phase of the cell-cycle (G0/G1, S, and G2/M) and in the sub-G1 peak was determined by flow cytometry. (B) PA-MSHA redistributed the cell cycle. Cell cycle distribution of (a) A549, (b) PC-9, and (c) NCI-H1975 cells in the four phases of the cell cycle are represented by percentages and representative graphs under these treatment conditions. ${ }^{*} \mathrm{P}<0.05$ for cells treated with PA-MSHA vs. the control group in all sub-G1, G0/G1, S and G2/M phases. PA-MSHA, Pseudomonas aeruginosa-mannose sensitive hemagglutinin.

indicating the proteolytic processing of the proenzyme to its active enzyme subunits. However, a concentration-dependent increase in cleaved caspase 8 was not observed when A549, PC-9, and NCI-H1975 cells were exposed to PA-MSHA. 


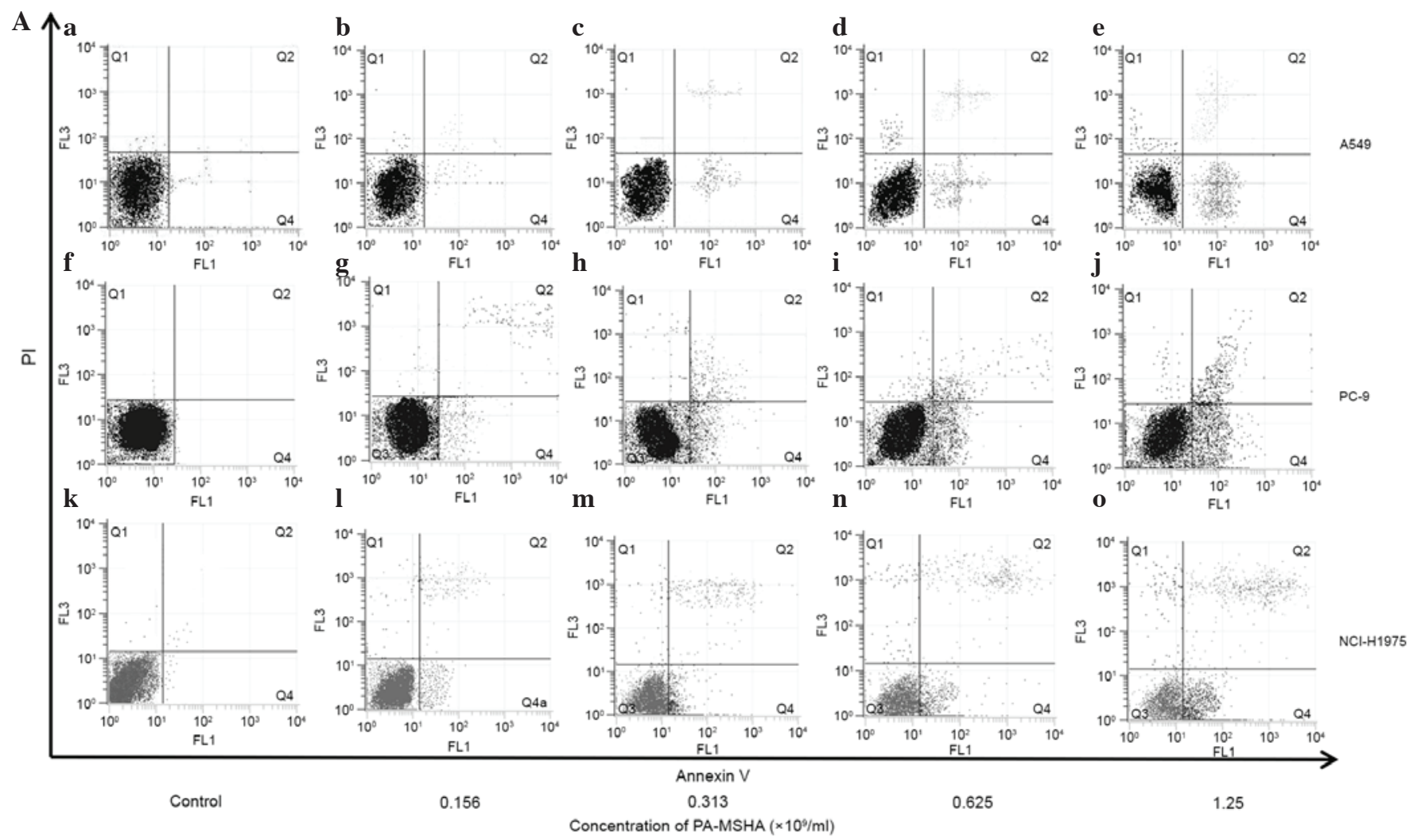

B

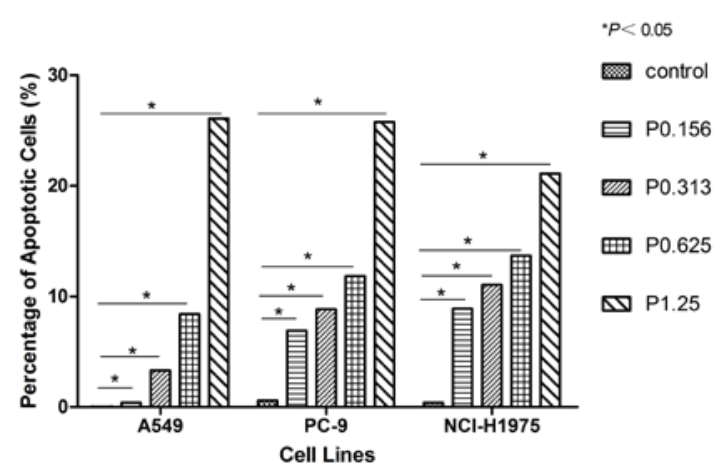

Figure 3. (A) Apoptotic fraction of cells detected by Annexin V staining/PI staining following different treatments. The ratio of apoptotic cells (Annexin V positive/PI negative) was measured in (a-e) A549, (f-j) PC-9, and (k-o) NCI-H1975 treated with control or the indicated concentrations of PA-MSHA for 24 h in serum-free medium. (B) The ratio of apoptotic cells (quadrant 2 plus quadrant 4 cells) was measured in A549, PC-9, and NCI-H1975 cells treated with phosphate-buffered saline, or the indicated concentrations of PA-MSHA, which are represented by percentages. * $\mathrm{P}<0.05$ vs. the control group. PI, propidium iodide; PA-MSHA, Pseudomonas aeruginosa-mannose sensitive hemagglutinin.

\section{Discussion}

For the last two decades, PA-MSHA has been used in China as an adjuvant therapy in the treatment of gastric cancer, breast cancer, lung cancer, and malignant lymphoma patients to reduce infection rates associated with chemotherapy, as well as to improve the sensitivity of chemotherapy, immune function, and quality of life $(23,24)$.

The present study examined the anti-cancer effects of PA-MSHA in vitro in three types of NSCLC cell lines, including two EGFR TKI-resistant lines. The results demonstrate that PA-MSHA inhibits cell proliferation, redistributes the cell cycle, and induces cell apoptosis in NSCLC cells with different genotypes in a concentration-dependent manner. The rate at which PA-MSHA inhibits proliferation was increased in PC-9,
A549 and NCI-H1975 cells compared with BEAS-2B cells. In addition, the effects of PA-MSHA on NSCLC were consistent with those previously observed in breast cancer cells expressing EGFR $(21,22)$. In previous studies by Liu et al $(21,22)$, PA-MSHA, which is characterized by mannose-sensitive hemagglutination pili, can specifically conjugate with the mannose-rich surface of high-mannose tumor cells. EGFR contains a large number of mannose oligosaccharides, which likely serve as the binding domain to PA-MSHA on the cell surface. In addition to these lectin-like activities, further studies have demonstrated that PA-MSHA can ablate the EGFR signaling cascade by reducing basal EGFR phosphorylation in breast cancer cells that overexpress EGFR (22). Growth suppression and cell death induced by PA-MSHA is mediated by the mannose-sensitive hemagglutination pili of $P$. aeruginosa 


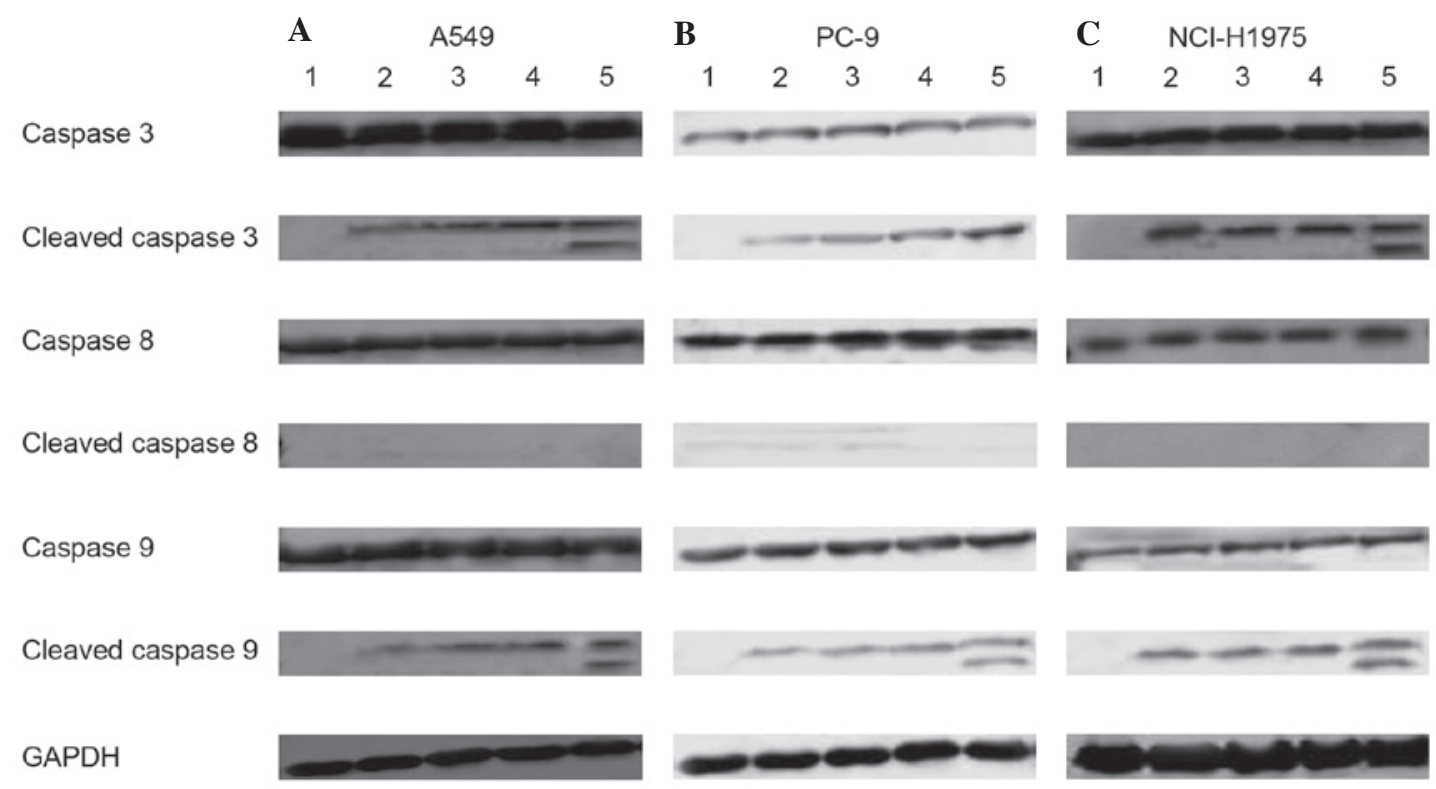

Figure 4. (A) A549 (B) PC-9 and (C) NCI-H1975 cells were treated with control or PA-MSHA at the indicated concentrations for 24 h. Each antibody was used in at least three independent experiments. Protein loading was normalized with GAPDH. Lane 1, control; lane 2, 0.156x 10\%/ml PA-MSHA; lane 3, $0.313 \times 10^{9} / \mathrm{ml}$ PA-MSHA; lane 4, $0.625 \times 10^{9} / \mathrm{ml} \mathrm{PA-MSHA;} \mathrm{lane} \mathrm{5,} 1.25 \times 10^{9} / \mathrm{ml}$ PA-MSHA. PA-MSHA, Pseudomonas aeruginosa-mannose sensitive hemagglutinin.

and that this effect may be independent of EGFR gene mutation status.

However, observed growth inhibition and cell death may be the result of apoptosis induced by cell cycle redistribution. Flow cytometric analysis and Annexin V-PI dual staining were performed to further investigate this. Consistent with results observed in breast cancer cell lines (21), a concentration-dependent decrease in the proportion of $\mathrm{S}$ phase cells and an increase in the sub-G1 population of the PA-MSHA-treated NSCLC cells was observed, suggesting that the arrested cells had entered into apoptosis. To the best of our knowledge, this is the first study to demonstrate PA-MSHA redistributes the cell cycle of NSCLC cells at the $\mathrm{S}$ and sub-G1 phases. Notably, proliferation inhibition, cell cycle arrest, and induction of apoptosis were observed in NSCLC cells treated with PA-MSHA independent of EGFR gene mutation status. This suggests that PA-MSHA-mediated effects may be a novel strategy to overcome the resistance to EGFR TKIs in NSCLC patients.

Earlier data has demonstrated that caspase-activated apoptosis is critical in the carcinogenesis, etiology, pathogenesis, and therapy of a number of human malignancies, including breast cancer, nasopharyngeal cancer and hepatocellular carcinoma $(20-22,25)$. This apoptosis was triggered by various stimuli, followed by initiation and execution via two predominant pathways, the intrinsic mitochondrial pathway and the extrinsic membrane death receptor pathway $(26,27)$. In the intrinsic mitochondrial pathway, caspase 9 and the downstream cleavage of caspase 3 was activated by the loss of mitochondrial membrane potential, which induces release of mitochondrial components into the cytoplasm, such as cytochrome $c$ (27). Alternatively, in the extrinsic pathway, the cell death ligands have been demonstrated to bind to cell surface death receptors and subsequently activate caspase 8 and caspase 3 (28).

In breast cancer, the data suggest that PA-MSHA triggered the extrinsic pathway, by interacting with the pro-apoptotic caspase cascade via the EGFR pathway, and the intrinsic apoptosis pathway, by mediating a mitochondrial effect $(21,22)$. However, the results of the present study suggest that apoptosis of NSCLC cell lines induced by PA-MSHA is mediated directly via caspase 3 and 9 and that the intrinsic pathway mediated by mitochondria may be important in apoptosis. Contrary to the results reported in breast cancer, the current study did not observe downregulated expression of caspase 8 and upregulated expression of cleaved caspase 8 in the three different NSCLC genotypes. These data suggest that the extrinsic pathway may not be key in PA-MSHA-induced NSCLC apoptosis. However, the dominant pathway requires further elucidation. The data from the present study suggest that PA-MSHA induced apoptosis of human NSCLC cells is mediated via caspase activation triggered by either mitochondrial or other pathways, such as EGFR-associated pathways.

In conclusion, the present study demonstrated, for the first time, that PA-MSHA treatment induces reduced proliferation, cell cycle redistribution and apoptosis via caspase family proteins in different NSCLC genotypes (PC-9, A549 and NCI-H1975 cells) independent of EGFR resistance. The in vitro experiments with PA-MSHA indicated that, in addition to an activated immune system, cytotoxicity may also contribute to NSCLC treatment. Treatment with PA-MSHA, either alone or in combination with standard therapeutic options, such as chemotherapy, radiotherapy, and particularly, targeted therapy, may be a novel strategy for the management of NSCLC. Further research is required to support the in vivo findings of the current study.

\section{Acknowledgements}

The present study was supported by the Shanghai Science and Technology Committee Natural Science Foundation of 
Shanghai (grant no. 12ZR 1406400), National Natural Science Foundation of China (grant no. 81302009), and Clinical Research Funds of Wu Jieping Medical Foundation (grant no. 320.6750.14278).

\section{References}

1. Siegel R, Naishadham D and Jemal A: Cancer statistics, 2012. CA Cancer J Clin 62: 10-29, 2012

2. Siegel R, Desantis C, Virgo K, Stein K, Mariotto A, Smith T, Cooper D, Gansler T, Lerro C, Fedewa S, et al: Cancer treatment and survivorship statistics, 2012. CA Cancer J Clin 62: 220-241, 2012.

3. Harari PM, Allen GW and Bonner JA: Biology of interactions: Antiepidermal growth factor receptor agents. J Clin Oncol 25: 4057-4065, 2007.

4. Hynes NE and Lane HA: ERBB receptors and cancer: The complexity of targeted inhibitors. Nat Rev Cancer 5: 341-354, 2005.

5. Fukuoka M, Wu YL, Thongprasert S, Sunpaweravong P, Leong SS, Sriuranpong V, Chao TY, Nakagawa K, Chu DT Saijo N, et al: Biomarker analyses and final overall survival results from a phase III, randomized, open-label, first-line study of gefitinib versus carboplatin/paclitaxel in clinically selected patients with advanced non-small-cell lung cancer in Asia (IPASS). J Clin Oncol 29: 2866-2874, 2011.

6. Mendelsohn J and Baselga J: Status of epidermal growth factor receptor antagonists in the biology and treatment of cancer. J Clin Oncol 21: 2787-2799, 2003.

7. Engelman JA and Jänne PA: Mechanisms of acquired resistance to epidermal growth factor receptor tyrosine kinase inhibitors in non-small-cell lung cancer. Clin Cancer Res 14: 2895-2899, 2008.

8. Li D, Ambrogio L, Shimamura T, Kubo S, Takahashi M, Chirieac LR, Padera RF, Shapiro GI, Baum A, Himmelsbach F, et al: BIBW2992, an irreversible EGFR/HER2 inhibitor highly effective in preclinical lung cancer models. Oncogene 27: 4702-4711, 2008.

9. Hirsch FR, Varella-Garcia M, Bunn PA Jr, Di Maria MV, Veve R, Bremmes RM, Barón AE, Zeng C and Franklin WA: Epidermal growth factor receptor in non-small-cell lung carcinomas: Correlation between gene copy number and protein expression and impact on prognosis. J Clin Oncol 21: 3798-3807, 2003.

10. Takezawa K, Okamoto I, Tanizaki J, Kuwata K, Yamaguchi H, Fukuoka M, Nishio K and Nakagawa K: Enhanced anticancer effect of the combination of BIBW2992 and thymidylate synthase-targeted agents in non-small cell lung cancer with the T790M mutation of epidermal growth factor receptor. Mol Cancer Ther 9: 1647-1656, 2010.

11. Miller VA, Hirsh V, Cadranel J, Chen YM, Park K, Kim SW, Zhou C, Su WC, Wang M, Sun Y, et al: Afatinib versus placebo for patients with advanced, metastatic non-small-cell lung cancer after failure of erlotinib, gefitinib, or both, and one or two lines of chemotherapy (LUX-Lung 1): A phase $2 b / 3$ randomised trial Lancet Oncol 13: 528-538, 2012.

12. Zhao M, Yang M, Ma H, Li X, Tan X, Li S, Yang Z and Hoffman RM: Targeted therapy with a Salmonella typhimurium leucine-arginine auxotroph cures orthotopic human breast tumors in nude mice. Cancer Res 66: 7647-7652, 2006.
13. Maletzki C, Linnebacher M, Kreikemeyer B and Emmrich J: Pancreatic cancer regression by intratumoural injection of live Streptococcus pyogenes in a syngeneic mouse model. Gut 57: 483-491, 2008.

14. Baban CK, Cronin M, O'Hanlon D, O'Sullivan GC and Tangney M: Bacteria as vectors for gene therapy of cancer. Bioeng Bugs 1: 385-394, 2010.

15. Zhao M, Geller J, Ma H, Yang M, Penman S and Hoffman RM: Monotherapy with a tumor-targeting mutant of Salmonella typhimurium cures orthotopic metastatic mouse models of human prostate cancer. Proc Natl Acad Sci USA 104: 10170-10174, 2007.

16. Crull $\mathrm{K}$ and Weiss S: Antibiotic control of tumor-colonizing Salmonella enterica serovar Typhimurium. ExpBiol Med (Maywood) 236: 1282-1290, 2011.

17. Hayashi K, Zhao M, Yamauchi K, Yamamoto N, Tsuchiya H, Tomita K and Hoffman RM: Cancer metastasis directly eradicated by targeted therapy with a modified Salmonella typhimurium. J Cell Biochem 106: 992-998, 2009.

18. Yam C, Zhao M, Hayashi K, Ma H, Kishimoto H, McElroy M, Bouvet $\mathrm{M}$ and Hoffman RM: Monotherapy with a tumortargeting mutant of S. typhimurium inhibits liver metastasis in a mouse model of pancreatic cancer. J Surg Res 164: 248-255, 2010.

19. Kreikemeyer B, Klenk M and Podbielski A: The intracellular status of Streptococcus pyogenes: Role of extracellular matrix-binding proteins and their regulation. Int J Med Microbiol 294: 177-188, 2004.

20. Wang J, Wu D and Chen L: Pseudomonas aeruginosa vaccine inhibits the proliferation of human nasopharyngeal cancer cells in vitro. Nan Fang Yi Ke Da Xue Xue Bao 32: 544-547, 2012 (In Chinese).

21. Liu ZB, Hou YF, Min-Dong, Di GH, Wu J, Shen ZZ and Shao ZM: PA-MSHA inhibits proliferation and induces apoptosis through the up-regulation and activation of caspases in the human breast cancer cell lines. J Cell Biochem 108: 195-206, 2009.

22. Liu ZB, Hou YF, Zhu J, Hu DL, Jin W, Ou ZL, Di GH, Wu J, Shen ZZ and Shao ZM: Inhibition of EGFR pathway signaling and the metastatic potential of breast cancer cells by PA-MSHA mediated by type 1 fimbriae via a mannose-dependent manner. Oncogene 29: 2996-3009, 2010.

23. Li Z, Hao D, Zhang H, Ren L, Yang Y, Li L, Chai J, Zhou X and Fu L: A clinical study on PA-MSHA vaccine used for adjuvant therapy of lymphoma and lung cancer. Hua Xi Yi Ke Da Xue Xue Bao 31: 334-337, 2000 (In Chinese).

24. Chen WD, Tang ZH and Xu F: Application of PA-MSHA vaccine adjuvant therapy and TAC scheme for treatment of breast carcinoma. Nan Fang Yi Ke Da Xue Xue Bao 29: 1204-1207, 2009 (In Chinese).

25. Philchenkov AA: Caspases as regulators of apoptosis and other cell functions. Biochemistry (Mosc) 68: 365-376, 2003.

26. Mathiasen IS and Jäättelä M: Triggering caspase-independent cell death to combat cancer. Trends Mol Med 8: 212-220, 2002.

27. Tretiakova I, Blaesius D, Maxia L, Wesselborg S, Schulze-Osthoff K, Cinatl J Jr, Michaelis M and Werz O: Myrtucommulone from Myrtus communis induces apoptosis in cancer cells via the mitochondrial pathway involving caspase-9. Apoptosis 13: 119-131, 2008.

28. Hegardt C, Andersson G and Oredsson SM: Different roles of spermine in glucocorticoid- and Fas-induced apoptosis. Exp Cell Res 266: 333-341, 2001. 